\title{
Robust Versus Optimal Rules in Monetary Policy: A Note
}

\author{
Maria Demertzis \\ and \\ Alexander F. Tieman
}




\title{
IMF Working Paper
}

European Department

\section{Robust Versus Optimal Rules in Monetary Policy: A Note}

Prepared by Maria Demertzis and Alexander F. Tieman ${ }^{1}$

Authorized for distribution by Emmanuel van der Mensbrugghe

June 2004

\begin{abstract}
This Working Paper should not be reported as representing the views of the IMF. The views expressed in this Working Paper are those of the author(s) and do not necessarily represent those of the IMF or IMF policy. Working Papers describe research in progress by the author(s) and are published to elicit comments and to further debate.

We provide a framework for analyzing the choice between optimal and robust monetary policy rules in the presence of paradigm uncertainty. We first discuss the conditions on uncertainty that render a robust rule preferable to an optimal rule. Second, we show how the degree of risk aversion of the policymaker increases the region in which the robust rule is preferred.

JEL Classification Numbers: E52, E58, C70

Keywords: Monetary Policy, Model Uncertainty, Policy Rules

Authors’ E-Mail Addresses: $\underline{\text { m.demertzis@dnb.nl, atieman@imf.org }}$

\footnotetext{
${ }^{1}$ Maria Demertzis is with De Nederlandsche Bank (the Dutch central bank). Alexander Tieman is with the IMF. The authors would like to thank Lex Hoogduin, Peter van Els, Ralph Teixeira, and Nicola Viegi for comments and helpful discussions. The views expressed in this paper do not necessarily reflect those of De Nederlandsche Bank or the European System of Central Banks.
} 


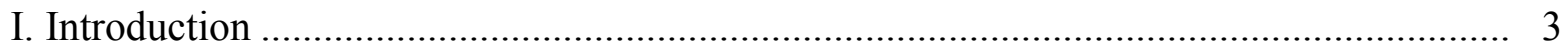

II. Optimal versus Robust Rules: A Model............................................................ 3

III. Policy Rules and Risk Preferences ............................................................... 6

A. Losses with Risk Neutrality ................................................................ 6

B. Losses with Risk Aversion ...................................................................... 7

IV. Discussion / Conclusions ........................................................................... 9

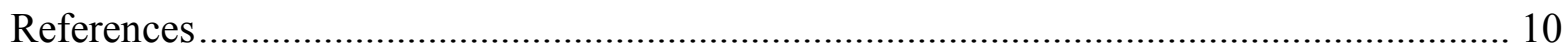

Figures

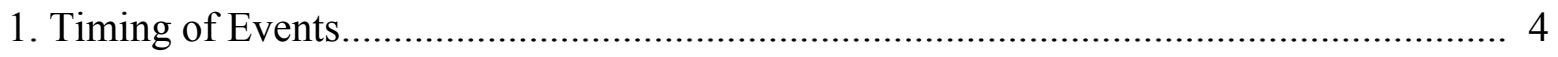

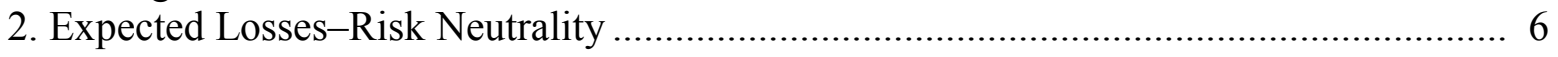

3. Utility with Risk Aversion ........................................................................... 8 


\section{INTRODUCTION}

Often the problem of model uncertainty in an optimization framework is dealt with by weighing the optimal outcomes with the respective probabilities in the form of an appropriately adjusted utility function (Holtham and Hughes Hallett, 1992; Frankel and Rockett, 1988). Alternatively, and if the losses from applying the wrong rule are considerable, policymakers often prefer to use rules that, although not optimal given the true economic model, perform relatively well across the spectrum of different possible models (Levin and others, 2001). Robustness is, therefore, associated with the wish to reduce the risks of applying a totally inappropriate rule. It is not immediately obvious what these rules should look like. Holtham and Hughes Hallett (1992) consider a weighted average of all underlying optimal rules. Gerdesmeier and others (2002) argue that '... in the face of paradigm uncertainty [robust rules] are derived from procedures that maintain the distinctiveness of the two paradigms and yet integrate analysis of the losses that rules give in each of the paradigms.'

In this paper we do not concern ourselves with the nature of robust rules. Instead, we provide for two issues: First, given the existence of at least one robust rule, we show for which levels of uncertainty one should apply the robust rule and when to revert back to using the optimal rules. Section III.A demonstrates this for the case of risk neutral preferences. Second, we show that the desire to apply a robust rule is linked to risk aversion such that as the latter increases, the likelihood of applying a given robust rule also increases (section III.B).

\section{OPTIMAL VERSUS ROBUST RULES: A MODEL}

Assume a central bank (CB) that optimizes the following loss function:

$$
\min _{r} L=E\left(\mathbf{x}^{\prime} \mathbf{Q} \mathbf{x}\right)
$$

\section{s.t.M}

with respect to its instrument, the interest rate $r$, and subject to the economic model prevailing, $\boldsymbol{M}$. Vector $\boldsymbol{x}$ represents the objectives, inflation and output, as deviations from their targets, and $\boldsymbol{Q}$ is a preference matrix of the central bank with the weights on the diagonal and zeros everywhere else. Function $L$ is continuous and twice differentiable, and $\boldsymbol{M}$ is a system of equations that define the constraining model. The optimization procedure produces a rule with respect to the instrument $r$ :

$$
r(.)=\arg \min L \text {. }
$$

We assume symmetric information between the central bank and the private sector. The system is characterized by uncertainty in the sense that a number of different models are candidates to explain the economy ex post. Which economic model will, in fact, prevail, however, is not known to the central bank. Suppose therefore, that the economy operates either as model $\boldsymbol{M}=\boldsymbol{A}$ or as model $\boldsymbol{M}=\boldsymbol{B}$ and, as we deal with a static world, no learning takes place. If model $\boldsymbol{A}$ is 
assumed, the optimal rule is $r_{A}$; if however, model $\boldsymbol{B}$ is assumed to be correct, then the optimal rule is $r_{B}$. The question that arises is which rule to apply given the uncertainty of which model will prevail. The sequence of events is such that the CB needs to take a decision as to which rule to implement first, and only after is the true model of the economy actually revealed.

Figure 1: Timing of Events

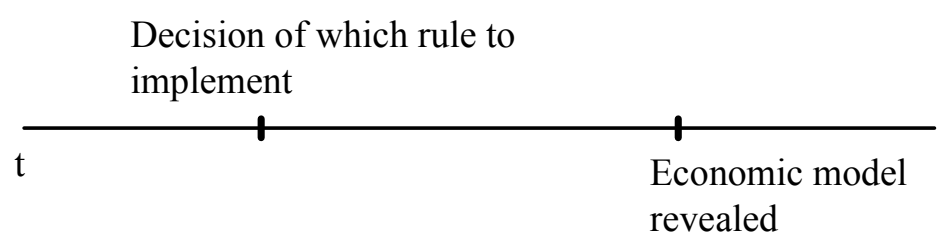

This implies, therefore, that the decision may actually turn out to be the wrong one. In a world where the economy can be described by only two alternative models, four different outcomes can therefore occur ex post:

- $\quad r_{A}$ rule is implemented and $\boldsymbol{A}$ occurs, incurring losses $L_{A, A} \geq 0$

- $\quad r_{A}$ rule is implemented and $\boldsymbol{B}$ occurs, incurring losses $L_{B, A}>L_{A, A}$

- $\quad r_{B}$ rule is implemented and $\boldsymbol{B}$ occurs, incurring losses $L_{B, B} \geq 0$

- $\quad r_{B}$ rule is implemented and $\boldsymbol{A}$ occurs, incurring losses $L_{A, B} \geq L_{B, B}$.

We suppose model $\boldsymbol{A}$ occurs with ex ante probability $q$ and model $\boldsymbol{B}$ with ex ante probability $(1-q)$. This probability $q$ is fixed and exogenously given, but unknown to the central bank. Then, under risk neutrality, the disutility of applying each of the rules is given by the expected losses:

$$
\begin{aligned}
& U_{r_{A}}(q)=\mathrm{E}\left[L\left(r_{A}\right)\right]=q L_{A, A}+(1-q) L_{B, A} \\
& U_{r_{B}}(q)=\mathrm{E}\left[L\left(r_{B}\right)\right]=q L_{A, B}+(1-q) L_{B, B}
\end{aligned}
$$

Naturally, the decision as to which one to implement is based on which of the two rules produces the lowest expected losses. The central bank will thus need to form a view on $q$ and apply policies accordingly. This, however, leaves the possibility of having applied the wrong rule, incurring ex post losses $L_{A, B}$ or $L_{B, A}$. And if these losses happen to be sufficiently large, then having applied the correct rule ex post becomes much more imperative. This is the reason that policymakers often search for alternative rules, which, although not optimal given the true economic model that actually prevails, have nevertheless the property of performing reasonably well across the spectrum of possible models. Assuming that such a rule exists, say $r_{C}$, there are two possibilities: 
- $\quad r_{C}$ rule is implemented and $\boldsymbol{A}$ occurs, incurring losses $L_{A, C}>L_{A, A}$

- $\quad r_{C}$ rule is implemented and $\boldsymbol{B}$ occurs, incurring losses $L_{B, C}>L_{B, B}$.

The disutility of using rule $r_{C}$ is now

$$
U_{r C}(q)=\mathrm{E}\left[L\left(r_{C}\right)\right]=q L_{A, C}+(1-q) L_{B, C}
$$

Rule $r_{C}$ will be implemented only if, for some probabilities, $r_{C}$ produces smaller losses than both $r_{A}$ and $r_{B}$. We label this property robustness in the given probability space.

We denote $q^{*}$ the probability for which the central bank is indifferent between applying $r_{A}$ or $r_{B}$.

Definition 1: A rule $r_{C}$ is considered robust vis-à-vis any optimal rules when two conditions hold:

1. Evaluated at $q^{*}, U_{r_{C}}\left(q^{*}\right)<U_{r_{A}}\left(q^{*}\right)=U_{r_{B}}\left(q^{*}\right)$, and

2. $\left|U_{r_{C}}^{\prime}(q)\right|<\left|U_{r_{A}}^{\prime}(q)\right|$ and $\left|U_{r_{C}}^{\prime}(q)\right|<\left|U_{r_{B}}^{\prime}(q)\right| \quad \forall \quad q \in[0,1]^{2}$

The first condition ensures that there is a range of probabilities for which it is optimal to apply $r_{C}$ and the second that the losses incurred are relatively invariant to the degree of uncertainty by comparison to the other two rules. Figure 2 provides a graphical representation of the definition ${ }^{3}$ and plots the expected losses of the three rules in terms of the unknown probability $q$.

2 Note that $U_{r_{c}}^{\prime}$ is short for $\frac{d U_{r_{C}}}{d q}$. Similarly for the other rules.

${ }^{3}$ Figure 2 refers to the case when risk neutrality is assumed. Our definition, however, holds irrespective of risk preferences. 
Figure 2: Expected Losses - Risk Neutrality

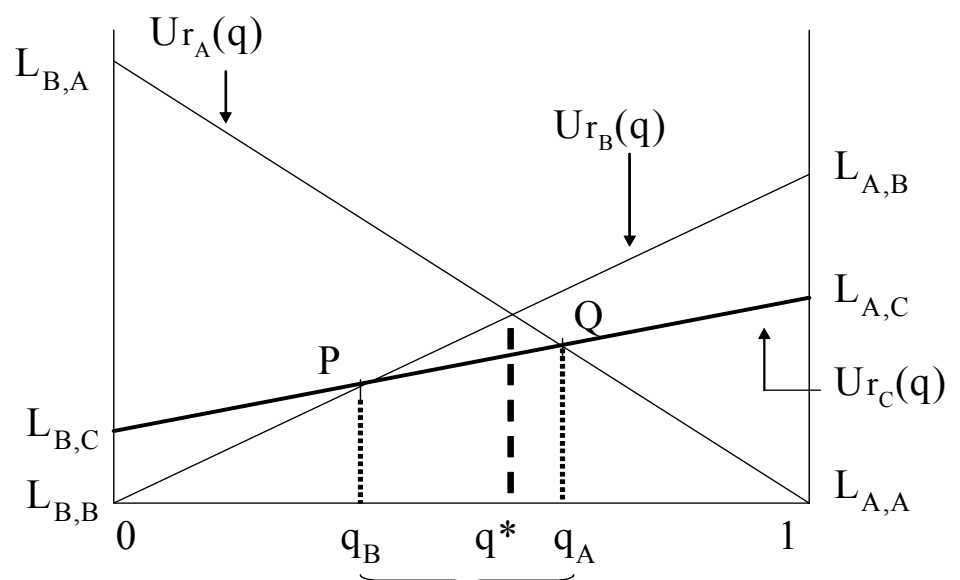

It follows that, for a robust rule, the following hold:

$$
\begin{aligned}
& L_{A, A}<L_{A, C}<L_{A, B} \\
& L_{B, B}<L_{B, C}<L_{B, B},
\end{aligned}
$$

and, from the second condition of the definition we have that

$$
\begin{aligned}
& \left|L_{A, C}-L_{B, C}\right|<\left|L_{B, A}-L_{A, A}\right| \\
& \left|L_{A, C}-L_{B, C}\right|<\left|L_{A, B}-L_{B, B}\right| .
\end{aligned}
$$

In what follows, we provide a framework that links the desirability of robust rules to the policymaker's degree of risk aversion.

\section{POLICY RULES AND RISK PREFERENCES}

\section{A. LOSSES WITH RISK NEUTRALITY}

Without loss of generality, we normalize $L_{A, A}=L_{A, A}=L_{B, B}=0$. Furthermore, the value of $q$ for which the central bank is indifferent between $r_{C}$ and $r_{A}\left(r_{B}\right)$ is $q_{A}\left(q_{B}\right)$. Looking again at Figure 2, we note that from equations (1) and (2) we have $U_{r_{A}}^{\prime}=C_{A}<0$ and $U_{r_{B}}^{\prime}=C_{B}<0$. The derivative $U_{r_{C}}^{\prime}$ is also constant, but its sign may be positive, negative, or zero, depending on which model, $\boldsymbol{A}$ or $\boldsymbol{B}$ is more favorable to rule $r_{C}$. Note that the range $\left(q_{B}, q_{A}\right)$ in Figure 2 shows the range of ex ante probabilities for which it is worth applying the robust rule $r_{C}$. The 
decision, therefore, of which rule to apply under risk neutrality is given by the following scheme:

$$
\begin{array}{llll}
0 \leq q<q_{B} & U_{r_{A}}(q)>U_{r_{C}}(q)>U_{r_{B}}(q) & \Rightarrow & \text { apply } r_{B} \\
q_{B}<q \leq q^{*} & U_{r_{A}}(q) \geq U_{r_{B}}(q)>U_{r_{C}}(q) & \Rightarrow & \text { apply } r_{C} \\
q^{*} \leq q<q_{A} & U_{r_{B}}(q)>U_{r_{A}}(q)>U_{r_{C}}(q) & \Rightarrow & \text { apply } r_{C} \\
q_{A}<q \leq 1 & U_{r_{B}}(q)>U_{r_{C}}(q)>U_{r_{B}}(q) & \Rightarrow & \text { apply } r_{A},
\end{array}
$$

while at $q_{A}\left(q_{B}\right)$ the CB is indifferent between $r_{A}\left(r_{B}\right)$ and $r_{C}$.

\section{B. EX ANTE LOSSES WITH RISK AVERSION}

Disutility $U .(q)$ in the previous section is a linear function of probability $q$, reflecting risk neutrality on the part of the central bank. We consider next the case when the central bank is risk averse and label the risk-averse disutility of the central bank as $U .(q)$, a non-linear function of $q$. The definition of risk aversion implies that $U_{r_{A}}(q), U_{r_{B}}(q)$ and $U_{r_{C}}(q)$ are now concave in the $(\mathbf{U}, \mathbf{q})$ space, such that, in comparison with risk neutrality, losses increase with uncertainty ${ }^{4}$. The properties of the functions are therefore summarized as

$$
\begin{array}{ll}
U_{r_{i}}(q)>U_{r_{i}}(q) & \text { for } q \in(0,1) \\
U_{r_{i}}(q)=U_{r_{i}}(q) & \text { for } q=(0,1) \\
U_{r_{i}}^{\prime \prime}(q)<0 & \text { for } q \in(0,1)
\end{array}
$$

for $i=A, B, C$. In relation to the robust rule $r_{C}$, it is also the case that

$$
\begin{aligned}
& U_{r_{A}}(q)-U_{r_{A}}(q)>\theta_{r_{C}}(q)-U_{r_{C}}(q) \\
& U_{r_{B}}(q)-U_{r_{B}}(q)>U_{r_{C}}(q)-U_{r_{C}}(q)
\end{aligned}
$$

which holds $\forall q \in(0,1)$ and follows from equations (4) and (5). This is necessarily the case because, for a given level of risk aversion, the degree of concavity is an increasing function of the distances $\left(L_{A, B}-L_{A, A}\right),\left(L_{B, A}-L_{B, B}\right)$, and $\left(L_{C, A}-L_{C, B}\right)$ for each of the rules, respectively. This reflects the range of losses across the probability spectrum when each rule is applied to either of the two models. By definition, this is less for the robust rule.

\footnotetext{
${ }^{4}$ Note that uncertainty increases the closer $q$ is to $1 / 2$.
} 
Proposition 1: The higher the degree of risk aversion, the larger the range of probabilities for which the central bank will choose to apply the robust rule.

Proof 1: Figure 3 plots the functions $U_{r_{i}}(q)$ and $U_{r_{i}}(q)$ for $i=A, B, C$.

Figure 3: Utility with Risk Aversion

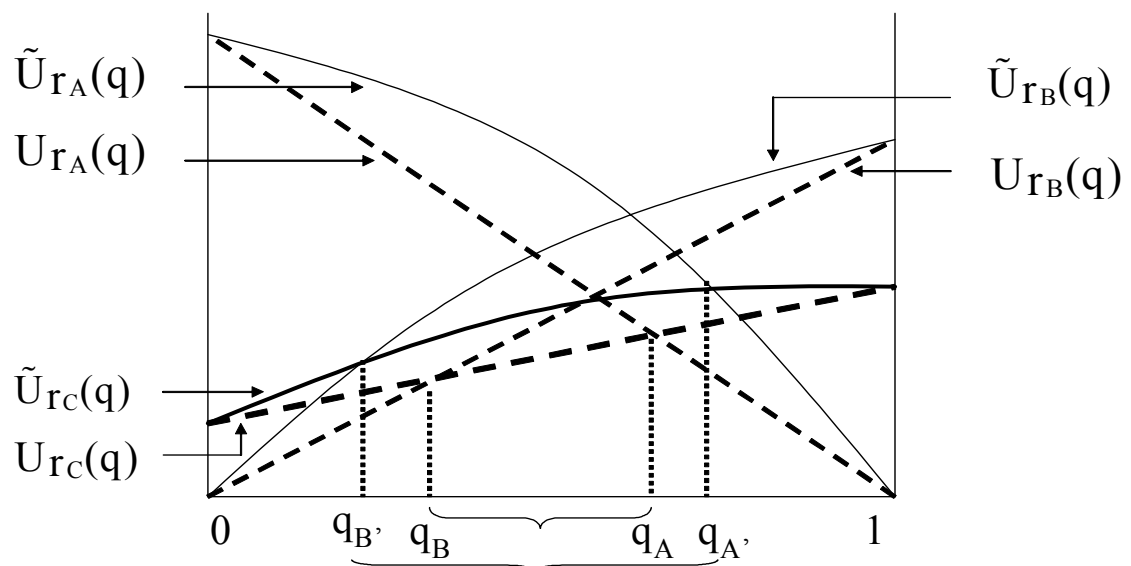

It suffices to show that (for continuous functions), if

$$
\begin{aligned}
& U_{r_{B}}(q)-\theta_{r_{C}}(q)>0, \text { for } q \geq q_{B}, \text { and } \\
& U_{r_{A}}(q)-\theta_{r_{C}}(q)>0, \text { for } q \leq q_{A},
\end{aligned}
$$

then $U_{r_{B}}(q)$ and $U_{r_{c}}(q)$ cross to the left of $q_{B}$ and $\theta_{r_{A}}(q)$ and $U_{r_{c}}(q)$ cross to the right of $q_{A}$. We show that (8) holds and, therefore, (9) will hold by analogy. We rewrite the left-hand side of (8) as follows

$$
U_{r_{B}}(q)-U_{r_{C}}(q)=\left[U_{r_{B}}(q)-U_{r_{B}}(q)\right]-\left[U_{r_{C}}(q)-U_{r_{C}}(q)\right]+\left[U_{r_{B}}(q)-U_{r_{C}}(q)\right]
$$

But we know that

$$
\begin{aligned}
& U_{r_{B}}(q)-U_{r_{C}}(q) \geq 0, \text { for } q \geq q_{B} \text { (by definition) and } \\
& U_{r_{B}}(q)-U_{r_{B C}}(q)>U_{r_{C}}(q)-U_{r_{C}}(q) \forall q \text { from (6). }
\end{aligned}
$$

Therefore, since $U_{r_{B}}\left(q_{B^{\prime}}\right)=U_{r_{B}}\left(q_{B^{\prime}}\right)$, it follows that $q_{B^{\prime}}<q_{B}$ and inequality (8) holds. Similarly we can show that $q_{A^{\prime}}<q_{A}$ and therefore, $q_{A}-q_{B}<q_{A}^{\prime}-q_{B}^{\prime}$. This implies that the 
robust rule $r_{C}$ will produce lower ex ante expected losses for a greater spectrum of probabilities $^{5}$. Furthermore, as the degree of risk aversion increases, so does the concavity of the utility functions, increasing the discrepancy between the magnitudes in (6) and (7), and, hence, increasing the range for which $r_{C}$ is preferred ${ }^{6}$.

\section{DISCUSSION/CONCLUSIONS}

Our objective was to show that the search for robust rules is dependent on the expected losses from applying optimal rules to incorrect models. We also show that this becomes even more eminent for risk averse policymakers. Our analysis does not provide an indication as to whether such rules exist or what their features would be ${ }^{7}$. Instead, we aim to formalize the comparison between robust and optimal rules and demonstrate how the former become more desirable as preferences become more risk averse.

\footnotetext{
${ }^{5}$ The same holds when the losses of applying the wrong rule are larger, i.e. if $L_{B, A}$ and $L_{A, B}$ increase.

${ }^{6}$ Note that for risk-loving policymakers, the results turn on their head and a robust rule becomes attractive for a smaller probability range compared to when they are risk indifferent. In other words, $q_{A}-q_{B}>q_{A}^{\prime}-q_{B}^{\prime}$ (proof by analogy).

${ }^{7}$ For further information on what the properties of robust rules should be, see Rudebusch and Svensson (1999) and Batini and Haldane (1999), among others.
} 


\section{REFERENCES}

Batini, Nicoletta, and Andrew Haldane, 1999, "Forward-looking Rules for Monetary Policy," in John B. Taylor, (ed), Monetary Policy Rules, University of Chicago Press, Chicago.

Frankel, J.A., and K. E. Rockett, 1988, "International Macroeconomic Policy Coordination when Policy Makers do not Agree on the True Model," American Economic Review, Vol. 78, No. 3, pp. 318-40.

Gerdesmeier, Dieter, Roberto Motto, and Huw Pill, 2002, "Paradigm Uncertainty and the Role of Monetary Developments in Monetary Policy Rules," paper presented at the Workshop on Monetary Policy Rules organized by the European Central Bank in Frankfurt on 11-12 March 2002.

Holtham, Gerald, and Andrew Hughes Hallett, 1992, "International Macroeconomic Policy Coordination When Policymakers Do Not Agree on the True Model: Comment," American Economic Review, Vol. 82, No. 4, pp. 1043-1051.

Levin, Andrew, Volker Wieland, and John C. Williams, 2001, "The Performance of ForecastBased Monetary Policy Rules Under Model Uncertainty," ECB Working Paper Series, No. 68.

Rudebusch, G., and Lars Svensson, 1999, “Policy Rules for Inflation Targeting," in John B. Taylor, (ed), Monetary Policy Rules, University of Chicago Press, Chicago. 